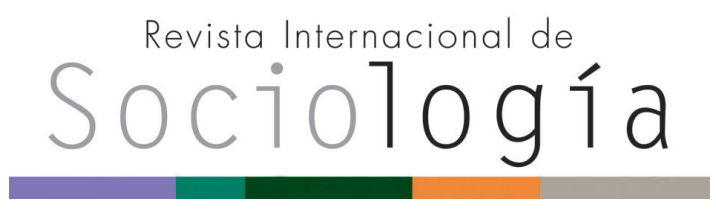

Revista Internacional de Sociología RIS

vol. 74 (3), e039, julio-septiembre, 2016, ISSN-L:0034-9712

doi: http://dx.doi.org/10.3989/ris.2016.74.3.039

\section{"NO ES UNA CRISIS, ES QUE YA NO TE QUIERO". Humor y protesta en el movimiento $15 \mathrm{M}$}

\author{
EDUARDO ROMANOS \\ Universidad Complutense de Madrid, España \\ eromanos@ucm.es
}

Cómo citar este artículo / Citation: Romanos, E. 2016. '"No es una crisis, es que ya no te quiero'. Humor y protesta en el movimiento 15M". Revista Internacional de Sociología 74 (3): e039. doi: http://dx.doi.org/10.3989/ris.2016.74.3.039

\section{“IT'S NOT A CRISIS, I JUST DON'T LOVE YOU ANYMORE". Humor and protest in the $15 \mathrm{M}$ movement}

Copyright: @ 2016 CSIC. Este es un artículo de acceso abierto distribuido bajo los términos de la licencia Creative Commons Attribution (CC BY) España 3.0.

Recibido: 13/10/2014. Aceptado: 01/07/2015. Publicado on line: 22/08/2016

\title{
Abstract
}

This article explores the relationship between humor and protest through a study of the strategic use of humor in the Spanish indignados movement. By focusing on four forms of communication (placards, performances, internal documents and Internet communications) in Madrid in 2011, the article assesses the subversive potential of humor in the communication of demands, the internal organization of the movement, the recruitment of potential activists and the construction of a collective identity. At the theoretical level, the article draws attention to the benefits that arise from combining an expressive focus in the analysis of humor with another which sees this as an instrumental action of those involved. Results show that the Spanish indignados were to a large degree aware of a number of benefits associated with the use of humor that went beyond having fun and, therefore, organized several activities in order to obtain these benefits.

\section{KeYWORDS}

Palabras Clave

Acción Social; Estrategia; Movimientos Sociales.

Social Action; Social Movements; Strategy. 


\section{INTRODUCCIÓN}

Si están indignados, ¿por qué se ríen tanto? Quien se hubiera acercado a la Puerta del Sol o a cualquiera de las otras plazas ocupadas en 2011 por el movimiento $15 \mathrm{M}$ se habría podido hacer esta pregunta. Aparentemente, es una de las paradojas de este movimiento: la combinación de la indignación contra "Ios políticos y los banqueros" con divertidas acciones y protestas. Por lo general, los medios de comunicación han subrayado el componente de indignación. De hecho, así se les conoce a nivel internacional: "los indignados". La etiqueta hace referencia al best-seller Indignez-vous! de Stéphane Hessel, que algunos identificaron como una fuente principal de inspiración de las protestas españolas. Los activistas, sin embargo, prefieren llamarse "movimiento $15 \mathrm{M}$ " en honor a la fecha de la manifestación de protesta que significó su pistoletazo de salida: el 15 de Mayo de 2011. La indignación es, sin duda, una emoción central en este movimiento. Este artículo trata de mostrar hasta qué punto las emociones asociadas al humor también lo son.

Los estudios sobre el humor configuran un campo interdisciplinar en el que, sin embargo, la atención prestada por parte de psicólogos, filósofos o lingüistas destaca en comparación con los científicos sociales (Kuipers 2006; Reay 2007). En el área específica de los movimientos sociales, la relevancia alcanzada por el estudio de las emociones en las últimas dos décadas (véase Goodwin, Jasper y Polletta 2001; 2004) no se ha traducido en un interés particular hacia el humor como, por otra parte, sí parece haberlo hecho en áreas más o menos afines, como la historia social (Hart 2007). Dentro de los escasos trabajos dedicados al humor y la protesta social destacan los centrados en el potencial subversivo del humor político en contextos represivos y no-democráticos (e.g., Pi-Sunyer 1977; Brandes 1977; Kenney 2003; Romanienko 2007; Merziger 2007; Davies 2007; Lewis 2008; Sorensen 2008; Oushakine 2012; Mersal 2011). En cualquier caso, la relación entre humor y protesta social no ha recibido mucha atención en la literatura sobre movimientos sociales (Kutz-Flamembaum 2014).

Este artículo explora la relación entre humor y protesta a través del estudio de la utilización del humor en el movimiento 15M. Para ello se centra en diversas iniciativas desarrolladas durante las primeras fases del movimiento en Madrid, epicentro de las protestas. La pregunta que guía la investigación es: ¿hasta qué punto el humor es un elemento meramente expresivo en el movimiento $15 \mathrm{M}$ o, por el contrario, también podemos hablar del humor como una acción instrumental de los activistas? En la respuesta a esta pregunta, el artículo matiza la perspectiva psicológica convencional (Apter 1991; Martin 2007) al subrayar cómo los "indignados" españoles fueron en buena medida conscientes de una serie de beneficios asociados a la utilización del humor que iban más allá de la propia acción de divertirse y, en consecuencia, organizaron diversas iniciativas con el objetivo de alcanzar esos beneficios. Obviamente, esto no quiere decir que el componente expresivo desaparezca. Simplemente se pretende subrayar con ello el componente instrumental del humor, que en general ha sido infravalorado en la literatura especializada. Algunos trabajos recientes apuntan en esta dirección (Roy 2007; Kutz-Flamenbaum 2007; Wettergren 2009). Este artículo se enmarca dentro de esta tendencia.

El artículo se abre con una breve introducción que sirve para situar el humor en relación con el estudio de la protesta y los movimientos sociales. Le sigue una breve discusión sobre la distinción weberiana entre acción expresiva e instrumental y su aplicación al ámbito del humor. A continuación se analiza la utilización del humor en el movimiento $15 \mathrm{M}$ a través de cuatro formas diferentes de comunicación: carteles, performances, documentos internos y la comunicación en internet. Los principales resultados de la investigación se recogen en un apartado final de conclusiones. Las fuentes analizadas en este artículo incluyen documentos, listas de correo y páginas web, material recopilado a partir de la observación participante y diez entrevistas individuales semiestructuradas a informantes clave del movimiento. La técnica de observación participante se aplicó en el contexto de las principales acciones de protesta organizadas por el movimiento, diversas reuniones (asambleas generales y temáticas) y otros acontecimientos de diversa naturaleza que tuvieron lugar en Madrid entre mayo y octubre de 2011. Las entrevistas fueron realizadas en Madrid y Barcelona entre octubre de 2011 y agosto de 2012. Nueve entrevistas fueron cara a cara y una por teléfono. En ocasiones las entrevistas se vieron acompañadas de comunicaciones posteriores mantenidas por correo electrónico. La mitad de los entrevistados eran mujeres y la otra mitad hombres. Las entrevistas recabaron información organizativa, la interpretación de determinados procesos y dinámicas del movimiento y otras cuestiones relacionadas según fueron surgiendo en el curso de la conversación.

\section{HUMOR, PROTESTA Y MOVIMIENTOS SOCIALES}

El humor es un fenómeno eminentemente social, susceptible de surgir en cualquier tipo de interacción (Martin 2007: 2). En términos generales, el humor se "refiere a todo aquello que la gente dice o hace y que se percibe como divertido, provocando que otros rían, así como al proceso mental activado en la creación y la percepción del estímulo divertido y la respuesta afectiva involucrada en su disfrute" (ibid., 5) ${ }^{1}$. El humor es, por tanto, no solo un proceso cognitivo sino también emocional. De hecho, puede entenderse como una emoción en sí misma (para un debate 
sobre esta cuestión, véase por ejemplo Hart 2007). Sin embargo, algunos psicólogos prefieren distinguir el humor de la emoción que provoca su percepción y que han venido a llamar apreciación humorística (Weisfeld 1993), alegría (Ruch 1993) o júbilo (Martin 2007). A su vez, esta emoción, como en general todas las emociones, tiene un componente expresivo, en este caso la risa y la sonrisa, que la hace visible a los demás. La universalidad de estas expresiones denota su carácter innato. Aunque cada cultura define qué aspectos son susceptibles de risa y en qué momentos podemos expresarla, todos nos reímos más o menos de la misma forma y podemos reconocer cuándo se están riendo los demás.

La protesta no ha sido un ámbito ajeno a la risa. La relación entre humor y protesta social hunde sus raíces en la historia, como han demostrado los trabajos de historiadores sociales, de las mentalidades y de la cultura popular. Hart (2007) destaca cuatro formas específicas de esta relación en el pasado. Durante siglos, los carnavales han simbolizado un mundo al revés en el que, durante un tiempo limitado y conocido, los miembros de las clases bajas podían expresar hostilidad hacia la oligarquía dominante. Los charivari eran manifestaciones sonoras del descontento de una parte de la comunidad normalmente dirigidas hacia matrimonios ilegítimos entre sus miembros pero que, en ocasiones, como señaló Tilly (1986), podían derivar en protestas políticas contra las autoridades (véase también Thompson 1991: cap. 8). Los bufones disfrutaban de una licencia especial para criticar públicamente al monarca, que afirmaba su poder absoluto ofreciéndoles protección oficial (véase Otto 2001). Por último, las publicaciones satíricas han conseguido sobrevivir a la censura apelando al imaginario popular y evitando mostrar de forma explícita el objeto de su burla.

Estas formas ritualizadas de expresión del descontento son anteriores al surgimiento de los movimientos sociales. En su desarrollo, los movimientos han incorporado algunas de ellas, adaptándolas al nuevo contexto, como en el caso de los desfiles carnavalescos o las performances burlescas, al mismo tiempo que han innovado nuevos usos subversivos del humor. En términos generales, el humor parece ser un elemento cada vez más visible e importante en el surgimiento y la continuidad de la protesta; una tendencia que parece relacionarse con el proceso de "estetificación" adicional de los movimientos sociales observado por Tucker (2010). El desarrollo de una esfera estética relacionada con el placer, el juego y la creatividad en la que cosmovisiones, experiencias e identidades alternativas encuentran un canal privilegiado de expresión ha tenido implicaciones importantes para la dinámica de los movimientos sociales a lo largo de la historia. Sin embargo, la dimensión transgresora de la aesthetic politics ha ido ganando preeminencia en el contexto de los recientes desafíos planteados desde los movimientos sociales a la interpretación neoliberal de la globalización (Tucker 2010: 11-12). El movimiento alter-globalización y la reciente ola transnacional de protesta corroborarían esta observación. Estos movimientos y movilizaciones se caracterizan no solo por un discurso específico sino también por un estilo rompedor e imaginativo en el que la diversión y la risa desempeñan un papel importante (Flesher Fominaya 2014). En este sentido, Shepard (2011) sitúa un "giro tragicómico" en la llegada de una nueva generación de activistas (véase también Boyd 2002).

Por otra parte, la creciente presencia del humor en los movimientos sociales parece responder asimismo al desarrollo de las nuevas tecnologías de la información y la comunicación. En distintas épocas el acceso a nuevos medios de comunicación ha abierto también la puerta a nuevas formas de relación entre el humor y la protesta. Por ejemplo, la popularización del soporte impreso contribuyó a la difusión de los dibujos cómicos a través de los grabados, panfletos y periódicos. De la misma manera, internet y las redes sociales representan hoy en día una plataforma con enormes posibilidades para el desarrollo de formas humorísticas de comunicación potencialmente subversivas (Van Laer y Van Aelst 2010).

En la literatura sobre movimientos sociales, los todavía escasos estudios dedicados a la relación entre humor e internet se han centrado en la técnica conocida como culture jamming (véase Wettergren 2009; Van Laer y Van Aelst 2010) en la que los activistas modifican el discurso publicitario original alterando visualmente los símbolos de las multinacionales (logos, eslóganes, etc.), dotándolos de un nuevo significado (Juris 2008). El culture jamming no nace con internet $^{2}$. Tampoco es - ni siquiera hoy en día- una técnica exclusivamente digital. Sin embargo, como señalan Van Laer y Van Aelst (2010), esta técnica ha crecido exponencialmente con el desarrollo de las nuevas tecnologías de la información y la comunicación. A su vez, la expansión del culture jamming ha traído consigo la del humor en la protesta. El humor es un elemento central en el culture jamming (Wettergren 2009), e internet ha facilitado su utilización en el desarrollo de esta estrategia simbólica. Los culture-jammers han abrazado el humor al mismo tiempo que "reconocen la capacidad de internet para hacer de la creación de parodias de anuncios algo mucho más fácil y suministrar una plataforma para que sus campañas y creaciones artísticas alcancen una audiencia mucho más amplia e internacional" (Van Laer y Wan Aelst 2010: 1159; véase también Woodside 2001; Klein 2002; Meikle 2002).

\section{EL HUMOR Y LA DIMENSIÓN EXPRESIVA- INSTRUMENTAL DE LA ACCIÓN SOCIAL}

La pregunta que guía esta investigación -hasta qué punto el humor fue una acción meramente expresiva o también instrumental en el movimiento 
$15 \mathrm{M}$ - remite a la clásica tipología weberiana de la acción social, que distingue entre una acción "racional con arreglo a fines" (zweckrational) de otra "racional con arreglo a valores" (wertrational) (Weber 1964: 20-21). Según Weber, ambas acciones son racionales y deliberadamente planeadas (en contraposición a la acción social no-racional, en cualquiera de sus modalidades: afectiva o tradicional). La acción racional con arreglo a fines viene "determinada por expectativas en el comportamiento tanto de objetos del mundo exterior como de otros hombres, y utilizando esas expectativas como 'condiciones' o 'medios' para el logro de fines propios racionalmente sopesados y perseguidos". La acción racional con arreglo a valores está "determinada por la creencia consciente del valor -ético, estético, religioso o de cualquier otra forma como se le intérprete- propio y absoluto de una determinada conducta, sin relación alguna con el resultado, o sea puramente en méritos de ese valor". La primera es instrumentalmente racional en relación con los fines, los medios y los resultados secundarios -tenidos todos racionalmente en cuenta y valorados- mientras que la segunda lo es solo en relación con la elección de medios. Así, el sentido de la acción racional con arreglo a valores "no se pone en el resultado, en lo que está fuera de ella, sino en la acción misma en su peculiaridad".

A partir de la tipología weberiana, algunos de los primeros estudios sobre movimientos sociales establecieron una distinción entre movimientos instrumentales y expresivos ${ }^{3}$. Los movimientos instrumentales se orientarían hacia un cambio en el entorno (por ejemplo, la implementación de determinada política) mientras que en los movimientos expresivos el cambio se realizaría entre los propios activistas, convirtiéndose la participación en un objetivo en sí mismo (véase Klandermans 2004). De manera similar, la dimensión instrumental-expresiva se ha venido aplicando a las actividades llevadas a cabo por los activistas dentro de los movimientos sociales y a las motivaciones que llevan a los individuos a participar en ellos. Sin embargo, cuando hablamos de la dimensión instrumental-expresiva, deberíamos tener claro que nos referimos a tipos ideales de acción social ya que, como reconoció el propio Weber, "muy raras veces la acción, especialmente la social, está exclusivamente orientada por uno u otro de estos tipos" (1964: 21; énfasis en el original). De la misma forma, los movimientos sociales reúnen en sí mismos aspectos instrumentales y expresivos (Zolberg 1972; Jenkins 1983; Tarrow 2012 y 2013), cuyo peso relativo varía a su vez en el tiempo (Klandermans 2004). Además, las actividades que se llevan a cabo en el seno de los movimientos sociales son en parte expresivas y en parte instrumentales (véase Della Porta y Diani 2011) y las motivaciones que llevan a los individuos a participar en ellos son una mezcla de motivos instrumentales y expresivos (Walgrave, Van Laer, Verhulst y Wouters, n.d.).
A pesar del tiempo transcurrido desde que Weber estableció su tipología y de las matizaciones que esta ha recibido desde entonces, la discusión en torno a la dimensión instrumental-expresiva de la acción social sigue suscitando interesantes debates en las ciencias sociales ${ }^{4}$. Este artículo tiene por objetivo llamar la atención sobre la parte instrumental de la ecuación con respecto al humor. Tradicionalmente, el humor se ha entendido como una actividad cuyo fin principal es la diversión y el entretenimiento que provocan la actividad en sí misma. Según el psicólogo Michael Apter (1991), el estado mental lúdico asociado al humor es un modo "paratélico", es decir, sin un objetivo externo a la propia acción. Este estado mental lúdico varía en su duración en el tiempo, pero se entiende como la interrupción de otro estado "télico" más serio en el que la acción se orienta hacia la consecución de un objetivo. Sin embargo, otros psicólogos sí reconocen una "meta subyacente" en los intercambios humorísticos, aunque esta sea inconsciente (Martin 2007). Entre dichas metas destacan tres, que se corresponden a su vez con las principales teorías sobre el origen del humor: rebajar la tensión en situaciones que amenazan el bienestar ("teoría del alivio"), señalar violaciones de patrones racionalmente aprendidos y aceptados ("teoría de la incongruencia") y reforzar la unidad del grupo mostrando cierto grado de grandeza frente al objeto motivo de mofa ("teoría de la superioridad") (véase Meyer 2000; Billing 2005). A continuación se evalúa de qué forma las acciones y protestas de los indignados españoles consiguieron alcanzar estas y otras metas, y hasta qué punto lo hicieron respondiendo a una estrategia consciente y deliberada.

\section{LOS DIFERENTES USOS DEL HUMOR EN EL MOVIMIENTO 15M}

He seleccionado cuatro formas de comunicación en las que analizar la utilización del humor en el movimiento 15M: i) los carteles individuales y pancartas colectivas portados por los activistas en las protestas; ii) las actuaciones (performances) organizadas por grupos específicos con ocasión de algunas protestas y reuniones; iii) la comunicación interna a través de la redacción de actas y otros documentos dirigidos primordialmente -aunque no de forma exclusiva- a los participantes en el movimiento; y iv) la comunicación en internet, con una atención especial a los mensajes enviados desde cuentas de Twitter asociadas de alguna forma al movimiento. Esta selección no es exhaustiva, pero tampoco aleatoria. El humor ha surgido en otras interacciones y formas de comunicación, pero parece haberlo hecho de una forma especialmente visible en las aquí señaladas, sobre todo de cara a la dimensión instrumental sobre la que quiero hacer hincapié en este artículo. 


\section{Carteles individuales y pancartas colectivas}

Algunos participantes en las protestas organizadas por el movimiento $15 \mathrm{M}$ en 2011 traían de casa carteles escritos de su puño y letra. Otros los diseñaron y escribieron en el curso de las manifestaciones. En algunas protestas hubo, de hecho, un espacio especialmente dedicado a esta tarea. Según cuenta un participante en la manifestación del 15 de mayo de 2011 en Barcelona: "En el suelo se extendían decenas de pancartas en blanco. La gente estaba alrededor, en círculo; observaba con timidez. Había rotuladores en el suelo. Se trataba de que cada uno escribiera su lema y luego, entre todos, nos los lleváramos calle abajo" (Muñoz 2011: 37). Precisamente, el carácter DiY (Do it Yourself) de los carteles marca una diferencia con respecto a toda una tradición de activismo en el que los eslóganes están organizados y elegidos por unos pocos que representan al resto.

La frecuencia de los carteles "manuales" con mensajes humorísticos fue significativa en las protestas, y así lo recogieron algunos medios de comunicación (véase, por ejemplo, El País, 21 mayo 2011). Muchos de esos mensajes subvertían textos familiares, que incluían poemas (v.g., "Me gusta cuando votas porque estás como ausente", basado en la primera línea del Poema 15 de Veinte Poemas de Amor y una Canción Desesperada, de Pablo Neruda), eslóganes publicitarios (v.g., "Tu banco, y cada día el de más gente", basado en un juego de palabras donde "banco" no hace referencia a la entidad financiera sino al mobiliario urbano, con el cartel colocado encima de uno de ellos), películas y canciones (v.g., "Rebeldes sin casa", o "Lobby is in the air"), frases célebres (v.g., "Españoles, la política ha muerto", alteración de la pronunciada por Carlos Arias Navarro con motivo de la muerte de Franco) y el lenguaje de las señales públicas (v.g., "Disculpen las molestias... jestamos cambiando el mundo!") $)^{5}$.

La utilización de este tipo de mensajes supone de alguna forma una ruptura cognitiva. Esta ruptura ha sido analizada principalmente desde la teoría de la incongruencia (véase Morreall 1989; cf. Veale 2004), que sostiene que la gente se ríe al percibir algo -en este caso un texto- que les parece chocante, sorprendente, inesperado y extraño de una manera no-amenazante. En el caso que nos ocupa, los manifestantes realizan una intervención creativa que modifica el texto original, transformándolo en un texto incongruente, salvo que este se entienda utilizando un marco de referencia diferente al original (sea la poesía, la publicidad, la música, el cine, el discurso político o el lenguaje cotidiano). En este caso, el marco de referencia alternativo es la protesta. Por ejemplo, los activistas alteraron el mensaje publicitario de un banco -en este caso sin necesidad de alterar el contenido del mensaje original, solo su emplazamiento, colocando el mensaje encima de un banco en la calle- para denunciar el elevado precio de la vivienda y el creciente número de desahucios ante la imposibilidad de pagar la hipoteca. De esta manera, en la interpretación del mensaje intervienen dos marcos de referencia consistentes en sí mismos pero normalmente no relacionados e incluso incompatibles entre sí a través de un proceso mental que Koestler (1964) definió como "bisociación", gracias al cual podemos percibir la incongruencia humorística (Martin 2007).

La elaboración de mensajes ingeniosos y humorísticos no ha sido únicamente una acción individual y más o menos espontanea sino también colectiva e incluso organizada. El humor ha sido visible en carteles colectivos, como el portado en la cabecera de una marcha que recorrió el centro de Madrid el 24 de junio de 2011 con el eslogan "No es una crisis, es que ya no te quiero". La marcha cerraba un fin de semana especialmente emotivo en el que seis marchas de activistas llegaron a Madrid desde distintos puntos de España tras recorrer cientos de kilómetros a pie.

Además, algunos activistas han organizado talleres específicos para el desarrollo de la creatividad colectiva en la elaboración de carteles individuales o colectivos, sobre todo con motivo de grandes movilizaciones, como el día global de acción del 15 de octubre de 2011 o en el primer aniversario del movimiento el 12 de mayo de 2012. Estos talleres se han llevado a cabo por lo general en las plazas o en los centros sociales que han servido de lugar de reunión y que han suministrado diferentes recursos al movimiento 15M (véase Romanos 2013). Además, como en el caso del anterior movimiento alterglobalización, el $15 \mathrm{M}$ ha contado con la participación de publicistas y diseñadores gráficos en el movimiento (los llamados "creativos") para la elaboración de carteles humorísticos que, utilizando técnicas adquiridas en el desarrollo de su profesión, alteran códigos culturales del capitalismo como forma de crítica y subversión. Este tipo de activismo será tratado más en profundidad en el apartado dedicado al desarrollo de técnicas de culture jamming en internet.

\section{Performances irónicas}

Las protestas del 15M también incluían actuaciones irónicas organizadas por grupos específicos. En Madrid, uno de estos grupos fue el Grupo de Teatro 15 de Mayo, creado el 18 de mayo de 2011 por iniciativa de una joven actriz y que llegó a contar con unas treinta personas que asistían regularmente a sus asambleas. Sus participantes apenas se conocían entre sí con anterioridad, a diferencia de otros grupos que también han realizado performances (aunque más dramáticas) dentro del $15 \mathrm{M}$, como el grupo de teatro gestual que organizó un "grito mudo" masivo la noche del 20 de mayo de 2011. Algunos participantes en el Grupo de Teatro 15 de Mayo tenían experiencias previas en el arte de la dramatiza- 
ción, incluso en el teatro de protesta, por ejemplo, en sesiones de "teatro foro" en Madrid6.

Entre las performances realizadas por el Grupo de Teatro 15 de Mayo destacan dos: el "entierro de la democracia" y el "aeróbic asambleario". La primera es una actuación habitual en diversos movimientos sociales, incluido el movimiento no-global. El grupo la realizó en dos ocasiones: el 27 de mayo y el 11 de junio. Consistía en un cortejo fúnebre que recorría el lugar de las protestas liderado por varios activistas disfrazados de religiosos y, tras ellos, un ataúd con la democracia y un grupo que lloraba su muerte. El cortejo tenía varias paradas en las que se rezaban oraciones irónicas en nombre de los derechos y las libertades perdidos. Los falsos curas intentaban mantener un semblante serio mientras que los miembros del cortejo exageraban su tristeza, llegando al paroxismo. El "aeróbic asambleario" también se realizó dos veces: el 12 y el 19 de junio. Tenía lugar antes del comienzo de la asamblea general en la Puerta del Sol, cuando todavía se mantenía la acampada y las asambleas eran masivas. Un grupo de activistas ataviado con ropa de deporte hortera y esperpéntica tomaba la asamblea y, tras realizar una serie de ejercicios en los que se practicaban los distintos gestos utilizados en la asamblea, se liaba en una discusión ridícula; por ejemplo, la forma de reformar la cocina de uno de ellos.

Estas actuaciones sirvieron para rebajar los ánimos en momentos de gran estrés causado por la intervención de la policía o las disputas internas. Este tipo de impactos se ha estudiado principalmente desde la perspectiva de la teoría del alivio (Lefcourt 2001; Lefcourt y Martin 1986), que sostiene que la gente experimenta el humor y la risa porque sienten que haciéndolo rebajan los niveles de estrés. La primera representación del entierro de la democracia en Madrid coincidió con un día de fuerte represión de los activistas en Barcelona, que atrajo la inmediata solidaridad del resto de participantes en las acampadas. La segunda representación del funeral se organizó el día de la investidura del alcalde de Madrid, que terminó en duros enfrentamientos entre los activistas y la policía. Por su parte, el aeróbic asambleario surgió a raíz de los conflictos internos generados en la acampada sobre la decisión de mantenerla o abandonarla. La actuación se mofaba de los problemas asociados a la asamblea; por ejemplo, los continuos bloqueos y las interminables discusiones acerca de la organización interna del movimiento y la toma de decisiones en su seno. También tenía una función didáctica: la performance mostraba como se puede alcanzar un consenso que incluya elementos de todas las propuestas expresadas por los participantes.

En este caso, Sara B. (mujer, 33 años), miembro del Grupo de Teatro 15 de Mayo reconoce cómo el humor descansaba de nuevo en la incongruencia:
La gente se reía mucho porque no dejábamos de poner algo que hacemos cada día, que en ese momento era nuestra rutina, en otro contexto que no tiene nada que ver, que es superficial. El aeróbic es hacer ejercicio, culto al cuerpo, lo que quieras, pero no tiene nada que ver con el pensamiento; es todo lo contrario. Entonces es muy gracioso.

Preguntada por el objetivo de la performance, Sara sostiene que los participantes tenían la intención consciente de romper la tensión de la asamblea para facilitar una conexión emocional que devolviera la creatividad perdida en el movimiento:

De repente todo lo racional se relaja... Es verdad que muchas veces cuando se te queda todo en la cabeza se hace una bola y es mucho más difícil de afrontar, es más estresante... A nivel grupal, en las asambleas, cuando la gente ya está tan exigida, esa energía no es nada creativa, desde ahí no puede salir absolutamente nada. Salen cosas, pero muy crispadas... [Cuando] te quedas en un plano muy racional de repente te desconectas de tu cuerpo y de tus emociones, es como que [estos elementos] se separan, y esto es un error porque también tienen cosas que decir. Son como tres planos que tienen que estar juntos porque son parte de ti. Entonces, creo que el humor consigue un camino fácil para llegar a esa conexión ${ }^{7}$.

Asimismo, los miembros del Grupo de Teatro 15 de Mayo eran conscientes de las dificultades del humor a la hora de provocar una reacción que tuviera implicaciones fuera de los límites del movimiento: "Ha servido de distensión, es una herramienta, pero como protesta tiene que ser muy ácido para tener [un impacto]. A nosotros nos faltaba ese punto: para que provoque una reacción que lleve a la acción... tiene que ser muy ácido, muy incisivo, hiriente si cabe"8. En este sentido el humor corre el peligro de quedarse en una válvula de escape, que puede terminar inhibiendo la movilización (Zijderveld 1983; Hart 2007). De ahí que el grupo admitiera la importancia de no "endulzar" las acciones, intentando ser más críticos en su forma y contenidos ${ }^{9}$. Sin embargo, a juzgar por lo observado, sus performances raramente alcanzaron esa "acidez".

Los participantes reconocen además otros beneficios asociados al montaje de performances humorísticas, como por ejemplo, atraer la atención de los medios de comunicación y la identificación de los espectadores con el movimiento ${ }^{10}$. Según Meyer (2000), una de las funciones más importantes del humor en la comunicación es la identificación comunicador-audiencia, realzando la credibilidad del que habla y fomentando la cohesión del grupo (véase también Malone 1980; Graham, Papa y Brooks 1992). En el movimiento $15 \mathrm{M}$, la función de identificación se potenció conscientemente en actuaciones en las que los miembros del grupo se reían de sí mismos mediante la exageración de una situación o un comportamiento hasta el ridículo, en este caso, los 
continuos bloqueos en la toma de decisiones y las discusiones sin límite. El espectador se reconocía en esa situación o ese comportamiento, lo que a su vez fomentaba la capacidad de autocrítica (cfr. Stewart y Kreuz 2003).

\section{Comunicación interna}

El humor también estuvo presente en los documentos internos. Uno de los casos más notables han sido los documentos producidos por el World Extension Team (WET) de acampadasol. Esta comisión se creó en los primeros días de la acampada en Madrid con el objetivo de difundir y coordinar el movimiento de los indignados a nivel internacional ${ }^{11}$. El 17 de agosto de 2011, la comisión lanzaba el Proyecto Acta, una iniciativa consistente en redactar las actas de las asambleas de la comisión de una forma divertida, como si fueran pequeñas historias que incorporaban elementos de lo que sucedía alrededor de la asamblea. Por lo general, los nombres de los participantes se sustituían por pseudónimos que además de preservar la identidad de aquellos servían para crear personajes dentro de las historias ${ }^{12}$.

Con Proyecto Acta, los miembros del grupo buscaban de manera consciente el empleo de "otro lenguaje" que contribuyera en alguna medida a rebajar los costes del activismo relacionados con la fatiga:

Lo que nos interesa es que se lean ¿Qué es lo que se lee? Lo que se lee es lo que nos cuentan. Se leen los cuentos, no se leen las actas [...] Yo creo que tiene que ver en parte con el ambiente que se creó en la comisión. Es decir, hacer del humor parte de tu tono, quitarle ceremoniosidad o peso [a las cosas] [...] era una comisión en la que nos divertíamos y nos apetecía estar ahí, nos apetecía estar juntos y no era ni una obligación juntarnos ni estar sin dormir, sino que realmente nos lo pasábamos bien ${ }^{13}$.

Durante cuatro meses la iniciativa sirvió para que los miembros del grupo y otros participantes en el movimiento leyeran las actas con mayor interés. Diversos experimentos en psicología social y comunicación han demostrado cómo el humor favorece la recepción de mensajes, atrayendo el interés de la audiencia hacia el acto de la comunicación. El humor provoca por lo general una respuesta más positiva y, lo que es más, prestigia de alguna forma la imagen del comunicador, facilitando así que futuros mensajes sean también recibidos de una forma más positiva (Gruner 1970; Markiewicz 1974; Wanzer, BoothButterfield y Booth-Butterfield 1996). En este sentido, los mensajes intercambiados en la lista de correo de WET dan cuenta de la positiva recepción de la iniciativa: los miembros de la lista dicen haber sentido una "gran emoción" al leer las actas, calificando a las mismas de "extraordinario ejercicio lírico", como un "best-seller, [que] atrapa hasta el final", o como "de lo mejor del $15 \mathrm{M}$ post-acampada"14. Por otra parte, la utilización del humor en la redacción de las actas sirvió para que los miembros del grupo se involucrasen en una de las tareas más aburridas en el movimiento: escribirlas. El empleo de ese "otro lenguaje" contribuyó a transformar en juego lo que en principio se veía como un trabajo. En este sentido, la diversión asociada al juego se demostró útil en el desarrollo del grupo y el mantenimiento de los esfuerzos organizativos (véase Shepard 2011).

Proyecto Acta también ayudó a rebajar las tensiones internas dentro de la comisión al brindar una oportunidad para comunicar los posibles enfados o críticas de una forma menos conflictiva. El humor es precisamente una forma de comunicación particularmente útil en situaciones "en las que un tono más serio y directo tiene el peligro de resultar demasiado confrontacional, potencialmente embarazoso o arriesgado" (Martin 2007: 17; véase también Kane, Suls y Tedeschi 1977). No se trata solo de rebajar la tensión, como en el caso de las performances irónicas señaladas más arriba, sino de comunicar además una crítica sin que el nivel de tensión suba por ello. En WET, uno de los motivos de tensión era, por ejemplo, el no cumplimiento de los horarios, cuya situación se recoge de esta forma en una de las actas:

Empezamos la asamblea a las 20:52. Los científicos de la comisión han estado en la universidad hasta las 19:00 para poder llegar puntualmente a Sol a las 20:00. Esta hora de retraso implica una merma en su producción científica lo que nos sitúa un poco más lejos de descubrir el bosón de Higgs, partículas supersimétricas o nuevos hallazgos en teoría de cuerdas y agujeros negros y el beneficio para la humanidad que eso supondría. Ergo... esto no puede seguir pasando ${ }^{15}$.

Por último, Proyecto Acta sirvió para reforzar la cohesión interna dentro de la comisión a través de bromas que hacían referencia a códigos compartidos entre sus miembros. En ocasiones, dichos códigos se empleaban para ridiculizar las actuaciones o la posición de opositores externos (por ejemplo, la policía) y, lo que es seguramente más importante, en referencia a grupos dentro del movimiento con los que los miembros de WET mantenían discrepancias. Entre ellos destaca la comisión de "política a largo plazo", cuyas veleidades revolucionarias eran ridiculizadas como propias de illuminati. Las bromas incluían, por ejemplo, evitar la expresión "a largo plazo" en la redacción de las actas ${ }^{16}$. En este sentido, es interesante observar cómo estas bromas fueron de las más comentadas y mejor recibidas en la lista de correo de $\mathrm{WET}^{17}$.

Según Billing (2005), el ridículo es uno de los usos primarios del humor a pesar de no haber sido una de las formas de humor más estudiadas (en detrimento del humor benigno) (véase también Obadare 2009). La teoría de la superioridad señala cómo "la gente se ríe abiertamente o para sus adentros de otros porque sienten algún tipo de triunfo sobre ellos o alguna forma de superioridad sobre los mismos" (Meyer 2000: 
314; véase también Gruner 1997; Zillmann 1983). Clarificando quiénes son distintos y hostiles, y clarificando qué les hace distintos y hostiles (este aspecto es, a menudo, el motivo de la mofa), los miembros del grupo fortalecen a su vez su identidad colectiva y favorecen las relaciones internas. Obviamente, el coste de esta forma de humor es el distanciamiento de los no-afines al grupo (Holmes y Marra 2002). Este es uno de los aspectos contradictorios más visibles de las funciones interpersonales del humor: mejora la cohesión social pero excluye a los individuos del grupo ajeno. No solo eso, sino que en alguna medida también dificulta la incorporación de potenciales seguidores que en principio pueden no compartir los códigos creados dentro del grupo.

Las actas no fueron los únicos documentos producidos por WET en un tono humorístico. El 19 de diciembre de 2011, los miembros de la comisión se declaraban en huelga a través de un comunicado publicado en internet. El documento en sí no es especialmente gracioso, pero sí la acción de convocar una huelga. Según reconocen los propios activistas, en este caso recurrieron de manera consciente a la incongruencia para conseguir un impacto a través del humor: "Llamarlo huelga tiene el cortocircuito que queríamos crear... No sé si es humor o es absurdo eso de una comisión de una acampada que no tiene nombre, que no tiene presidente, que no tiene nada, convoca a la huelga"18. El objetivo era llamar la atención sobre un problema fundamental que ellos advertían en ese momento en el movimiento: "No hay prácticamente diálogo, no hay construcción colectiva, no hay espacios para encontrarnos todos y debatir y vibrar y plantarle cara a los de arriba" 19 . Según los activistas, el movimiento había llegado a esa situación debido a un concepto demasiado estricto del asambleísmo, que proponían desechar para funcionar a través de la confianza en lugar de la burocracia. En este caso, los activistas utilizaban el humor como forma de "clarificar" su mensaje (Meyer 2000). Llamando a la "huelga", los activistas condensaban sus argumentos en un titular atractivo, que servía para clarificar su posición. Sin embargo, como en el caso de las actas, la iniciativa no estuvo exenta de fuertes críticas por parte de un importante sector del movimiento.

\section{Comunicación en internet}

Los indignados españoles también han utilizado el humor en técnicas de culture jamming, muchas de ellas aplicadas a la comunicación en internet. Para ello han contado con la colaboración de los llamados "creativos" y radical community managers, profesionales que no se sitúan fuera del movimiento sino como parte del mismo, en ocasiones desempeñando un papel activo y central. Los "creativos" incluyen a todos aquellos diseñadores, ilustradores y otros profesionales o expertos de la publicidad o las artes vi- suales que han puesto sus conocimientos técnicos al servicio del movimiento (véase, por ejemplo, Mattoni 2012; Doerr y Mattoni 2014). Por su parte, los radical community managers han aplicado sus habilidades en la promoción de contenidos en internet para difundir los mensajes y las imágenes de los creativos al mismo tiempo que aportado otras técnicas de culture jamming, por ejemplo, la creación de cuentas falsas de Twitter que suplantan la identidad de determinadas personas y ridiculizan la actuación de la policía (v.g., @acampadapolicia, @putohelicoptero, @elestupadesol) y las autoridades (v.g., @desdelamoncloa, @espeonzaaguirre). Estas acciones pueden ser vistas como una actualización de la técnica conocida como "corrección de la identidad", una forma tradicional de parodia que sin embargo ha encontrado un nuevo impulso en acciones de activistas como The Yes Men, que "se hacen pasar por portavoces de multinacionales y gobierno para desvelar su 'verdadera' naturaleza y difundir con ello mensajes anticapitalistas" (Firat y Kuryel 2010: 10).

Como en otras formas subversivas del humor, la utilización de la técnica de "corrección de la identidad" se agudizó en momentos de gran tensión. Un ejemplo lo aporta la cuenta @acampadapolicia creada el 2 de agosto de 2011, una vez que la policía despejara la Puerta del Sol de manifestantes, cerrara el acceso a la misma y empleara la violencia contra los que intentaban entrar. Algunos activistas comenzaron a tuitear "como si la policía estuviera acampada dentro de la plaza, con sus problemáticas, sus celebraciones, sus exigencias, sus necesidades". La cuenta recibió una rápida y positiva respuesta. Entre los objetivos de la cuenta, uno de sus creadores reconoce la búsqueda consciente de una mayor resonancia: "Cuando empezaron a formarse todos los grupos de trabajo, todas las comisiones; cuando empezamos a debatir el consenso de mínimos, la permanencia [en la plaza] o no... la cosa se puso excesivamente seria". Contrarrestar la rigidez alcanzada por el movimiento fue otra de las razones alegadas por este radical community manager para participar en su creación:

sirve para difundir mucha información, de otra forma, con humor... Las risas dan megustas, dan comentarios y dan exponencialidad y se multiplican, y puedes comunicar con humor lo mismo que con un mensaje serio y llegar mucho más lejos ${ }^{20}$.

En definitiva, las nuevas tecnologías parecen potenciar funciones sociales del humor ya señaladas en actividades off-line, como la ridiculización de oponentes y la difusión no-confrontacional de la crítica, tanto externa como interna al movimiento. Como señaló Stéphane M. Grueso, otro activista muy activo en Twitter, internet también ha brindado una herramienta especialmente útil para rebajar la tensión en el seno del movimiento, no solo a nivel colectivo sino también individual: "Había una serie de momentos 
en los que asistía a una situación en la que me emocionaba mucho, o me enfadaba, y a la hora de tuitear intentaba poner una chanza más o menos acertada pero que me ayudara a procesar esas emociones" 21 .

\section{Conclusiones}

Este artículo subraya las ventajas de combinar en el análisis del humor un enfoque expresivo con otro que lo entienda como una acción instrumental por parte de los involucrados en la misma. Combinar ambos enfoques nos ayuda a entender mejor la compleja relación entre humor y protesta en los movimientos sociales. La utilización del humor en los movimientos sociales tiene sin duda un fuerte componente expresivo: los activistas lo utilizan porque haciéndolo se lo pasan bien. Este artículo subraya, sin embargo, cómo la utilización del humor en el movimiento $15 \mathrm{M}$ responde también y de una forma importante a razones estratégicas: los activistas han empleado el humor en sus protestas con la intención de lograr una serie de objetivos que van más allá de la propia diversión. Para ello han escrito mensajes humorísticos en carteles individuales y colectivos, organizado actuaciones irónicas, intentado cambiar el estilo de la comunicación dentro de los comités y empleado técnicas de "corrección de la identidad" en internet. Entre otros objetivos, estas iniciativas han perseguido: i) identificar problemas internos y externos al movimiento y llamar la atención de los participantes -reales y potenciales- sobre ellos; ii) rebajar los ánimos en momentos de gran estrés; iii) identificar a la audiencia con las demandas del movimiento; iv) rebajar los costes del activismo relacionados con la fatiga; v) comunicar los posibles enfados o críticas internas de una forma menos dramática y conflictiva; vi) reforzar la cohesión interna; y vii) ridiculizar a los oponentes. ¿Ha sido una estrategia colectiva, consciente e intencionada? Parece ser que sí, como demuestran la organización de talleres para la promoción de mensajes irónicos e imaginativos en los carteles, la búsqueda explícita de una conexión emocional entre el público por parte de los grupos encargados de organizar las performances, el desarrollo de iniciativas humorísticas específicas dentro de los comités, o la aplicación de habilidades y conocimientos específicos vinculados a técnicas publicitarias y de difusión de contenidos en internet.

El humor ha sido un elemento que ha permanecido en las acciones de los indignados españoles a pesar del relativo declive en términos de participación y apoyo social sufrido por el movimiento desde mayo de 2011 (Sandiumenge 2013). Este hecho denota de alguna forma la importancia y centralidad del humor dentro del mismo. Este artículo se ha centrado en algunas iniciativas desarrolladas en Madrid en los primeros meses del movimiento. Iniciativas posteriores incluyen la creación de la cuenta de Facebook Humor Indignado 99\%, con la que cerca de 600.000 de "los de abajo se ríen de los de arriba" (cifra de septiembre de 2014), o el lanzamiento de Mongolia, "revista satírica sin mensaje alguno" cuyos editores dicen en su página web perseguir "con tinta a bandoleros, farsantes, embusteros y demás fauna que anteponga sus intereses personales y los del Fondo Monetario Internacional a los del mundo mundial" -su tirada alcanza los 40.000 ejemplares, véase García de Blas (2012)-. Por otra parte, a finales de marzo de 2012 culture jammers de diversos países se dieron cita en Barcelona en unas "jornadas internacionales de activismo creativo" organizadas por el grupo Enmedio bajo el título Cómo Acabar con el Mal y con la mirada puesta en las movilizaciones convocadas con motivo del primer aniversario del $15 \mathrm{M}^{22}$. Las jornadas estaban dirigidas al $99 \%$ de la población y entre sus objetivos figuraban inventar modos creativos de intervención social, formar grupos de trabajo que realizarán intervenciones concretas a corto plazo y dotarse de herramientas creativas para la acción en la calle y en la red. Todo ello "con humor e ironía", en palabras de una de las organizadoras (citado en Simón 2012).

¿Hasta qué punto el humor es una novedad o una evolución dentro del sector de los movimientos sociales en España, y en concreto en Madrid? En su investigación sobre el movimiento antiglobalización de principios de la década de 2000 en Madrid, Flesher Fominaya (2007) señalaba cómo el humor fue una pieza clave a través de la cual los activistas consiguieron integrar miembros nuevos y conceptualizar acciones directas dirigidas al público en general. El humor también facilitó la creación de una identidad política nueva y distintiva, diferente de la izquierda institucional. La investigación de Flesher Fominaya también sostenía que el reconocimiento del potencial subversivo del humor en el activismo político era por entonces un concepto todavía relativamente novedoso en los círculos movimentistas españoles. Los diferentes usos del humor dentro del movimiento $15 \mathrm{M}$ analizados en este artículo permiten ver hasta qué punto ha cambiado el panorama. No todos los activistas se muestran favorables a la utilización del humor, pero su empleo generalizado en el movimiento ha contribuido a crear un estilo distintivo que le diferencia de otros movimientos y formas de acción colectiva, también dentro de la izquierda. Las innovaciones en los movimientos sociales, como en general en otros muchos ámbitos, son a menudo obra de las nuevas generaciones. El cambio de estilo de los activistas españoles, que a su vez podemos englobar en un cambio más amplio a nivel transnacional (Shepard 2011), parece no ser una excepción al verse impulsado por la llegada de una nueva generación de activistas. Los miembros de esta generación poseen diversas habilidades, entre otras, saber utilizar de manera estratégica las nuevas tecnologías de la información y la comunicación; herramientas que se han demostrado especialmente útiles en la elaboración y difusión de contenidos humorísticos. La 
intención de los actores no basta para explicar los resultados de su acción, que se ve condicionada por un amplio conjunto de factores externos a la misma (Flesher Fominaya 2014). Sin embargo, reconocer el uso estratégico que los activistas hacen del humor puede ayudarnos a explicar su creciente visibilidad y centralidad en los movimientos sociales. Los activistas son conscientes del impacto que el humor puede causar fuera del movimiento y lo utilizan en consecuencia.

Algunos psicólogos señalan cómo el humor ayuda a afirmar algunas ambivalencias acerca del yo, revelando tensiones internas y preocupaciones experimentadas por la persona (véase Murgatroyd 1991). A muchos indignados españoles no les duelen prendas en reconocer que participan en un movimiento provisional y en permanente construcción. De hecho, su apuesta por la deliberación se basa precisamente en una comunicación de calidad que sea capaz de transformar las preferencias individuales en aras del bien común (Romanos 2011). El reconocimiento de las ambivalencias e inseguridades a través del hu- mor puede ser un elemento más que lo diferencia de movimientos pasados más "serios". Aunque una discusión de este tipo entra fuera de los límites de este artículo, quizá no sea descabellado pensar que la percepción de una supuesta ruptura con la protesta "típica" en este sentido puede haber facilitado la participación en el nuevo movimiento de gente sin un historial pasado o reciente de activismo.

\section{Agradecimientos}

Este artículo está asociado al proyecto CSO201341035-P financiado por el Ministerio de Economía y Competitividad. Quiero agradecer a James Jasper, John Krinsky, Elizabeth Borland, Emily Campbell, Donatella della Porta, Doug McAdam, Jeff Goodwin, Sidney Tarrow, Robert Fishman, Benjamín Tejerina, Ignacia Perugorría, a los evaluadores anónimos de esta revista y a los asistentes a las conferencias y los seminarios donde presenté versiones anteriores de este artículo sus interesantes comentarios y sugerencias.

\section{Notas}

1. Esta y otras traducciones del inglés son obra del autor.

2. El término "culture jamming" lo acuña en 1984 en San Francisco la banda Negativland. Muchos observadores sitúan a la música punk, los Yippies norteamericanos, la Internacional Situacionista y al movimiento dadaísta entre sus precursores.

3. Por ejemplo, Gusfield (1963: 20-24) reconoce la diferencia al mismo tiempo que la matiza a través de la idea de "status movements". Véase también Rucht (1988).

4. Por ejemplo, Fishman (2009; véase también 2004; 2014) señala los beneficios derivados de combinar un enfoque instrumental en el análisis de las redes sociales con otro que las entienda como bienes en sí mismos.

5. Ejemplos tomados de Minchinela (2011).

6. El "teatro foro" es una técnica creada por el dramaturgo de origen brasileño Augusto Boal como parte de su Teatro del Oprimido que llama a la intervención activa y directa del público en la representación.

7. Esta cita y la anterior, extraídas de la entrevista realizada por el autor a S. B., 26 Abril 2012, Madrid.

8. Ibid.

9. Acta del 'Grupo de Teatro 15 de Mayo', 29 Junio 2011. Disponible en: https://n-1.cc/pg/groups/171971/teatroquince-de-mayo/ (acceso 21 Mayo 2012).

10. Acta del 'Grupo de Teatro 15 de Mayo' minutes, 13 Junio 2011. Disponible en: https://n-1.cc/pg/groups/171971/ teatro-quince-de-mayo/ (acceso 21 Mayo 2012).

11. La elección del nombre de la comisión no deja de tener su gracia. Las siglas WET son el contrapunto de DRY, las siglas de Democracia Real Ya: la plataforma digital creada con motivo del lanzamiento de la convocatoria de manifestación del 15 de mayo de 2011 y que con

miento de los indignados.
12. Actas de WET, 17 Agosto-14 Diciembre 2011. Disponibles en: https://n-1.cc/pg/groups/130782/solextension-estatal-e-internacional/ y http://actasmadrid. tomalaplaza.net/

13. Entrevista telefónica realizada por el autor a C. (8 Abril 2012).

14. Mensajes de correo, 3 y 6 Septiembre, 1 y 2 Diciembre 2011, lista de correo WET.

15. Acta de WET, 16 Noviembre 2011. Disponible en: https://n-1.cc/pg/groups/130782/sol-extension-estatale-internacional/ (Acceso 7 Marzo 2012).

16. Acta de WET, 30 Noviembre 2011. Disponible en: http://actasmadrid.tomalaplaza.net/ (acceso 7 Marzo 2012).

17. Mensajes de correo, 6 Septiembre 2011, lista de correo WET.

18. Entrevista telefónica realizada por el autor a C. (8 Abril 2012).

19. "Extensión Internacional de Sol se declara en huelga¿Somos el 99\%?", 19 Diciembre 2011. Disponible en: http://madrid.tomalaplaza.net/2011/12/19/ (acceso 22 Mayo 2012).

20. Entrevista de Stéphane M. Grueso a Zulo. Disponible en: http://www.15m.cc/2011/12/conversaciones-15mcczulo.html (acceso 15 Febrero 2012).

21. Entrevista del autor a S.M.G., 17 Octubre 2011, Madrid.

22. Entre los participantes figuraban el británico John Jordan -uno de los fundadores de Reclaim the Streets!-, los alemanes A.F.R.I.K.A Gruppe, los estadounidenses The Illuminator y los internacionales Yomango, además de varios españoles. 


\section{Referencias Bibliográficas}

Apter, M. J. 1991. "A Structural-Phenomenology of Play". Pp. 13-29 en Adult Play: A Reversal Theory Approach, editado por J. H. Kerr y M. J. Apter. Amsterdam; Berwyn, PA: Swets \& Zeitlinger.

Barker, C. 2001. "Fear, Laughter, and Collective Power: The Making of Solidarity at the Lenin Shipyard in Gdnask, Poland, August 1980". Pp. 175-194 en Passionate Politics: Emotions and Social Movements, editado por J. Goodwin, J. M. Jasper y F. Polleta. Chicago: The University of Chicago Press. http://dx.doi.org/10.7208/ chicago/9780226304007.003.0011

Billig, M. 2005. Laughter and Ridicule: Towards a Social Critique of Laughter. Londres: SAGE. PMCid:PMC2396390

Boyd, A. 2002. "Irony, Meme Warfare and the Extreme Costume Ball". Pp. 245-243 en From ACT UP to the WTO, editado por B. Shepard y R. Hayduk. Nueva York: Verso.

Brandes, S. H. 1977. "Peaceful Protest: Spanish Political Humor in a Time of Crisis". Western Folklore 36:331346. http://dx.doi.org/10.2307/1499197

Bruner, M. L. 2005. "Carnivalesque Protest and the Humorless State". Text and Performance Quarterly 25:136-155. http://dx.doi.org/10.1080/10462930500122773

Davies, C. 2007. "HumourandProtest: JokesunderCommunism”. International Review of Social History 52:291-305. http:// dx.doi.org/10.1017/S0020859007003252

della Porta, D. y M. Diani. 2011. Los Movimientos Sociales. Madrid: CIS-UCM.

Doerr, N. y A. Mattoni. 2014. "Alternative Media and Transnational Public Spaces". Pp. 386-405 en The Revolution Will Not Be Televised? Media and Protest Movements, editado por K. Fahlenbrach, E. Sivertsen and R. Werenskjold. Nueva York: Berghahn.

Firat, B. Ö. and A. Kuryel. 2011. "Introduction". Pp. 9-20 en Cultural Activism: Practices, Dilemmas and Possibilities, edited by B. Ö. Firat and A. Kuryel. Amsterdam: Rodopi.

Fishman, R. M. 2004. Democracy's Voices: Social Ties and the Quality of Public Life in Spain. Ithaca: Cornell University Press.

Fishman, R. M. 2009. "On the Costs of Conceptualizing Social Ties as Social Capital". Pp. 67-83 en Social Capital: Reaching Out, Reaching In, editado por V. O. Bartkus y J. Davis. Cheltenham: Edward Elgar. http://dx.doi. org/10.4337/9781848445963.00012

Fishman, R. M. 2014. "Networks and Narratives in the Making of Civic Practice". En Varieties of Civic Innovation, editado por C. Sirianni y J. Girouard. Nashville: Vanderbilt University Press.

Flesher Fominaya, C. 2007. "The Role of Humour in the Process of Collective Identity Formation in Autonomous Social Movement Groups in Contemporary Madrid". International Review of Social History 52:243-258. http://dx.doi.org/10.1017/S0020859007003227

Flesher Fominaya, C. 2014. Social Movements and Globalization. Nueva York: Palgrave Macmillan.

García de Blas, E. 2012. "España tiene una salida (Barajas)". El País, 27 Julio.

Ghonim, W. 2012. Revolution 2.0 The Power of the People Is Greater than the People in Power. Londres: Harper Collins. PMid:23229555 PMCid:PMC3561564

Goodwin, J., J. M. Jasper y F. Polleta. 2004. "Emotional Dimensions of Social Movements". Pp. 413-432 en
The Blackwell Companion to Social Movements, editado por D. A. Snow, S. A. Soule y H. Kriesi. Malden, MA: Blackwell. http://dx.doi.org/10.1002/9780470999103. ch18

Goodwin, J., J. M. Jasper y F. Polleta. 2001. Passionate Politics: Emotions and Social Movements. Chicago: The University of Chicago Press. http://dx.doi. org/10.7208/chicago/9780226304007.001.0001

Graham, E. E., M. J. Papa and G. P. Brooks. 1992. "Functions of Humor in Conversation: Conceptualization and Measurement". Western Journal of Communication 56:161-183. http://dx.doi. org/10.1080/10570319209374409

Gruner, C. R. 1970. "The effect of humor in dull and interesting informative speeches". Central States Speech Journal 21:160-166. http://dx.doi. org/10.1080/10510977009363017

Gruner, C. R. 1997. The Game of Humor: A Comprehensive Theory of Why We Laugh. New Brunswick, NJ: Transaction Publisher. PMCid:PMC21023

Gusfield, J. R. 1963. Symbolic Crusade: Status Politics and the American Temperance Movement. Urbana: University of Illinois Press.

Hart, M. 2007. "Humour and Social Protest: An Introduction". International Review of Social History 52:1-20. http:// dx.doi.org/10.1017/S0020859007003094

Holmes, J. and M. Marra. 2002. "Over the edge? Subversive humor between colleagues and friends". Humor 15:6587. http://dx.doi.org/10.1515/humr.2002.006

Holton, A. E. and S. C. Lewis. 2011. "Journalists, Social Media, and the Use of Humor on Twitter". The Electronic Journal of Communication 21.

Jenkins, J. C. 1983. "Resource Mobilization Theory and the Study of Social Movements". Annual Review of Sociology 9:527-553. http://dx.doi.org/10.1146/annurev.so.09.080183.002523

Juris, J. S. 2008. Networking futures : the movements against corporate globalization. Durham, N. C.: Duke University Press. http://dx.doi.org/10.1215/9780822389170

Kane, T. R., J. Suls and J. T. Tdeschi. 1977. "Humour as a Tool os Social Interaction". Pp. 13-16 en It's a Funny Thing, Humour, editado por A. J. Chapman y H. C. Foot. Oxford: Pergamon Press.

Kenney, P. 2003. A Carnival of Revolution. Princeton: Princeton University Press.

Klandermans, B. 2004. "The Demand and Supply of Participation: Social-Psychological Correlates of Participation in Social Movements". Pp. 360-379 en The Blackwell Companion to Social Movements, editado por D. A. Snow, S. A. Soule y H. Kriesi.

Klein, N. 2002. No Logo: No Space, No Choice, No Jobs. Nueva York: Picador.

Koestler, A. 1964. The Act of Creation. Londres: Hutchinson.

Kuipers, G. 2006. Good Humor, Bad Taste: A Sociology of the Joke. Berlin; Nueva York: Mouton de Gruyter.

Kutz-Flamembaum, R. V. 2007. "Code Pink, Raging Grannies, and the Missile Dick Chicks: Feminist Performance Activism in the Contemporary Anti-War Movement". NWSA Journal 19:89-105. http://dx.doi.org/10.2979/ NWS.2007.19.1.89

Kutz-Flamembaum, R. V. 2014. "Humor and Social Movements". Sociology Compass 8:294-304. http:// dx.doi.org/10.1111/soc4.12138 
Lefcourt, H. M. 2001. Humor: The Psychology of Living Bouyantly. Nueva York: Kluwer Academic. http://dx.doi. org/10.1007/978-1-4615-4287-2

Lefcourt, H. M. and R. A. Martin. 1986. Humor and Life Stress: Antidote to Adversity. Nueva York: Springer-Verlag. http://dx.doi.org/10.1007/978-1-4612-4900-9

Lewis, B. 2008. Hammer \& Tickle: The History of Communism Told Through Communist Jokes. Londres: Weidenfeld $\&$ Nicolson.

Malone, P. B. 1980. "Humor: A Double-edge Tool for Today's Managers". Academy of Management Review 5:357360. http://dx.doi.org/10.5465/AMR.1980.4288842 / http://dx.doi.org/10.2307/257110

Markiewicz,D.1974. “EffectsofHumoronPersuasion”. Sociometry 37:407-422. http://dx.doi.org/10.2307/2786391

Martin, R. A. 2007. The Psychology of Humor: An Integrative Approach. Amsterdam: Elsevier Academic Press.

Mattoni, A. 2012. Media Practices and Protest Politics: How Precarious Workers Mobilise. Aldershot: Ashgate. PMCid:PMC3461819

McKay, G. 1996. Senseless Acts of Beauty: Cultures of Resistance since the Sixties. Londres: Verso.

Meikle, G. 2002. Future active: media activism and the internet. Nueva York: Routledge.

Mersal, I. 2011. "Revolutionary Humor". Globalizations 8:669674. http://dx.doi.org/10.1080/14747731.2011.629030

Merziger, P. 2007. "Humour in Nazi Germany: Resistance and Propaganda? The Popular Desire for an All-Embracing Laughter". International Review of Social History 52:275290. http://dx.doi.org/10.1017/S0020859007003240

Meyer, J. C. 2000. "Humoras a double-edged sword: Fourfunctions of humor in communication". Communication Theory 10:310-331. http://dx.doi.org/10.1111/j.1468-2885.2000. tb00194.x

Minchinela, R. 2011. "Lemas y consignas del movimiento 15M". Consultado 12 de febrero de 2012 (https://vimeo.com/27147951)

Morreall, J. 1983. Taking Laughter Seriously. Albany: SUNY Press.

Muñoz, A. 2011. "Del síndrome Wikileaks a la democracia 2.0. Las redes sociales y el 15-M". Pp. 34-43 en Las voces del 15-M, editado por J. M. Antentas, A. FernándezSavater, A. Muñoz, A. Requena y E. Vivas. Barcelona: Los libros del lince.

Murgatroyd, S. 1991. "The Nature and Social Functions of Humor". Pp. 119-129 en Adult Play: A Reversal Theory Approach, editado por J. H. Kerr y M. J. Apter. Amsterdam; Berwyn, PA: Swets \& Zeitlinger.

Obadare, E. 2009. "The Uses of Ridicule: Humour, 'Infrapolitics' and Civil Society in Nigeria". African Affairs 108:241261. http://dx.doi.org/10.1093/afraf/adn086

Otto, B. K. 2001. Fools are Everywhere: The Court Jester around de World. Chicago: University of Chicago Press.

Oushakine, S. A. 2012. "Red Laughter": On Refined Weapons of Soviet Jesters". Social Research 79:189-216.

Pi-Sunyer, O. 1977. "Political Humor in a Dictatorial State: The Case of Spain". Ethnohistory 24:179-190. http://dx.doi. org/10.2307/481742

Reay, M. 2007. "Humor". En Blackwell Encyclopedia of Sociology, editado por G. Ritzer. Malden: Blackwell.

Romanienko, L. A. 2007. "Antagonism, Absurdity, and the Avant-Garde: Dismantling Soviet Oppression through the Use of Theatrical Devices by Poland's 'Orange' Solidarity Movement". International Review of
Social History 52:133-151. http://dx.doi.org/10.1017/ S0020859007003161

Romanos, E. 2011. "El 15M y la democracia de los movimientos sociales", Books and Ideas (18/11/2011), Dossier "Débats autour du 15M" (http://www.booksandideas. net/El-15M-y-la-democracia-de-los.html).

Romanos, E. 2013. "Collective Learning Processes within Social Movements: Some Insights into the Spanish 15-M/Indignados Movement". Pp. 203-219 en Understanding European Movements, editado por C. Flesher Fominaya y L. Cox. Londres: Routledge.

Roy, C. 2007. "When Wisdom Speaks Sparks Fly: Raging Grannies Perform Humor as Protest". Women's Studies Quarterly 35:150-164.

Ruch, W. 1993. "Exhilaration and Humor". Pp. 605-616 en Handbook of Emotions, editado por M. Lewis y J. M. Haviland. Nueva York: Guilford.

Rucht, D. 1988. Themes, Logics and Arenas of Social Movements: A Structural Approach. Greenwich, CT: JAI Press.

Sandiumenge, L. 2013. "El humor en tiempos de cólera". Público, 13 Mayo. Consultado 20 de mayo de 2013 (http://www.publico.es/455299/el-humor-en-tiemposde-colera).

Sandlin, J. A. 2008. "'Mixing pop (Culture) and politics'”: Cultural resistance, culture jamming, and anti-consumption activism as critical public pedagogy. Curriculum Inquiry 38:323-350. http://dx.doi.org/10.1111/j.1467873X.2008.00411.x

Shepard, B. H. 2011. Play, Creativity, and Social Movements: If I Can't Dance, It's Not My Revolution. Nueva York: Routledge.

Simón, P. 2012. "Creatividad y humor para tomarse el activismo en serio". Periodismo Humano, 8 Marzo. Consultado 4 de mayo de 2012 (http://periodismohumano.com/culturas/creatividad-y-humor-para-tomarse-el-activismoen-serio.html).

Sorensen, M. J. 2008. "Humor as a Serious Strategy of Nonviolent Resistance to Oppression". Peace \& Change 33:167-190. http://dx.doi.org/10.1111/j.14680130.2008.00488.x

Stewart, C. O. and R. J. Kreuz. 2003. "On the Communicative Functions of Exaggeration: How to be a Million Times Clearer". Communication Research Reports 20:331340. http://dx.doi.org/10.1080/08824090309388832

Tarrow, S. 2012. Strangers at the Gates: Movements and States in Contentious Politics. Cambridge: Cambridge University Press. http://dx.doi.org/10.1017/ CBO9780511920967

Tarrow, S. 2013. The Language of Contention: Revolutions in Words, 1688-2012. Cambridge: Cambridge University Press. http://dx.doi.org/10.1017/CBO9781139567190

Teune, S. 2007. "Humour as a Guerrilla Tactic: The West German Student Movement's Mockery of the Establishment". International Review of Social History 52:115-132. http://dx.doi.org/10.1017/S002085900700315X

Tilly, C. 1986. The Contentious French. Cambridge: The Belknap Press of Harvard University Press. http:// dx.doi.org/10.4159/harvard.9780674433984 PMCid:PMC213616

Thompson, E. P. 1991. Customs in Common. Londres: The Merlin Press, Ltd.

Tucker, K. H. 2010. Workers of the World, Enjoy! : Aesthetic Politics from Revolutionary Syndicalism to the Global Justice Movement. Philadelphia: Temple University Press. 
Van Laer, J. and P. Van Aelst. 2010. "Internet and Social Movement Action Repertoires: Opportunities and limitations". Information Communication \& Society 13:11461171. http://dx.doi.org/10.1080/13691181003628307

Veale, T. 2004. "Incongruity in humor: Root cause or epiphenomenon?" Humor 17: 419-428. http://dx.doi. org/10.1515/humr.2004.17.4.419

Walgrave, S., J. Van Laer, J. Verhulst and R. N. D. Wouters. 'Why People Protest. Comparing Demonstrators' Motivations Across Issues and Nations". Consultado 1 de agosto de 2012 (http://www.m2p.be/publications/1280218856.pdf).

Wall, D. 1999. Earth First and the Anti-Roads Movement: Radical Environmentalism and Comparative Social Movements. Londres: Routledge.

Wanzer, M., M. Booth-Butterfield and S. Booth-Butterfield. 1996. "Are Funny People More Popular: The Relationship of Humor Orientation, Loneliness, and Social Attraction". Communication Quarterly 44:42-52. http://dx.doi.org/10.1080/01463379609369999

Weber, M. 1964. Economía y sociedad. México: FCE.

EDUARDO ROMANOS es Investigador Ramón y Cajal en el Departamento de Sociología I de la Universidad Complutense de Madrid. Sus principales áreas de investigación son la sociología política y la sociología histórica, con un interés especial por los movimientos sociales y la protesta.
Weisfeld, G. E. 1993. "The Adaptative Value of Humor and Laughter". Ethology and Sociobiology 14:141-169. http://dx.doi.org/10.1016/0162-3095(93)90012-7

Wettergren, A. 2009. "Fun and Laughter: Culture Jamming and the Emotional Regime of Late Capitalism". Social Movement Studies 8:1-15. http://dx.doi. org/10.1080/14742830802591119

Wilkins, J. and A. J. Eisenbraun. 2009. "Humor Theories and the Physiological Benefits of Laughter". Holistic Nursing Practice 23:349-354. http://dx.doi.org/10.1097/ HNP.0b013e3181bf37ad / PMid:19901609

Woodside, S. 2011. Every Joke is a Tiny Revolution: Culture Jamming and the Role of Humour. University of Amsterdam.

Zijderveld, A. 1983. "Humour and Laughter in the Social Fabric". Current Sociology 31:37-57. http://dx.doi. org/10.1177/001139283031003005 / http://dx.doi. org/10.1177/001139283031003006

Zillmann, D. 1983. "Disparagement Humor". Pp. 85-107 en Handbook of Humor Research (vol. 1), editado por P. E. McGhee y J. H. Goldstein. Nueva York: Springer-Verlag. http://dx.doi.org/10.1007/978-1-4612-5572-7_5 